Research Article

\title{
Quantized Feedback Control of Active Suspension Systems Based on Event Trigger
}

\author{
Jinwei Sun $\mathbb{D}^{1},{ }^{1}$ Jingyu Cong $\mathbb{D},{ }^{2}$ Weihua Zhao, ${ }^{1}$ and Yonghui Zhang ${ }^{1}$ \\ ${ }^{1}$ School of Vehicle Engineering, Xi'an Aeronautical University, Xi'an 710077, China \\ ${ }^{2}$ Marine Resource Utilization in South China Sea and School of Information and Communication Engineering, \\ Hainan University, Haikou 570228, China \\ Correspondence should be addressed to Jingyu Cong; jingyuconghnu@126.com
}

Received 1 October 2020; Revised 12 April 2021; Accepted 3 May 2021; Published 12 May 2021

Academic Editor: Luca Landi

Copyright (C) 2021 Jinwei Sun et al. This is an open access article distributed under the Creative Commons Attribution License, which permits unrestricted use, distribution, and reproduction in any medium, provided the original work is properly cited.

\begin{abstract}
As the deviation error will accumulate during the data acquisition and transcoding process of an active suspension system, this paper presents a sliding-mode-based quantized feedback control method. The aim of the controller is to improve the vertical performance of vehicles in the presence of external interferences. A 7-DOF suspension model with nonlinear springs and actuator dynamics is built for the control purpose. Firstly, a static quantizer on the uplink channel and a dynamic quantizer on the downlink channel are considered in the sliding mode controller to reduce the cumulative error and suppress the sprung mass motions. Secondly, an event trigger mechanism is introduced in the controller design process to reduce energy consumption and operation frequency of the actuator. The overall stability of the designed controller is proved by the Lyapunov functions. Finally, numerical simulations are carried out to evaluate the efficacy of the proposed controller. Different quantitative and trigger conditions are discussed, and the random road excitation is considered as the external disturbance input. The results of the control method indicate that the designed controller can improve the riding comfort with little loss of handling stability compared with the passive system. In addition, the trigger mechanism can improve the working efficiency of actuators effectively.
\end{abstract}

\section{Introduction}

As the passive suspension system can hardly provide a better compromise between riding comfort and handling stability, the controllable suspension system has received extensive attention in recent years $[1,2]$, and the control technology can be classified into a semiactive and active suspension system [3]. Despite the high cost and power requirement, an active suspension system can provide desired control force and improve the vehicle's dynamic performance significantly [2].

Many active suspension control approaches such as optimal control [4], adaptive control [5], and linear quadratic regulator control have been proposed. An adaptive backstepping controller with hard constraints for the active suspension system is designed in [6] to stabilize the attitude of vehicle and improve ride comfort. A saturated adaptive robust controller to improve suspension performance is proposed in [7], while in [8], backstepping control to compensate actuator lag in the nonlinear full vehicle model is designed. As the accurate dynamic model of the suspension system is difficult to determine, many different control techniques have been proposed for active suspension systems. A fuzzy logic controller has been used to provide desired actuation force for the capacity of resisting external disturbances and parameter uncertainty [9]. An output feedback finite-time controller was designed in [10] for active suspension systems to compensate the unknown external disturbance and improve the suspension performance, and a bioinspired dynamics-based adaptive tracking active suspension controller was presented in [11] to cancel vibration energy transmitted by suspension inherent nonlinearity. Another well-known control method which can exert robustness on unmeasured disturbances and parameter uncertainties for nonlinear systems is the sliding mode control [12]. The traditional sliding mode scheme can hardly 
be implemented on complicated systems with unknown parameters and the system model must be known. Hence, many research studies focused on the combination of control algorithms for uncertain systems to achieve better performance [13]. An enhanced adaptive self-organizing fuzzy sliding mode controller for an active suspension system is proposed in [14], and a hybrid self-organizing fuzzy and radial basis-function neural-network controller is used to reduce the sprung acceleration in [15]. The modelfree adaptive sliding controller proposed in [16] uses a functional approximation and online learning technique to improve the control performance.

With respect to the existing results, most of conventional control algorithms tend to ignore the performance degradation caused by quantization errors, which may cause a certain deviation from the original data after decoding at the receiving end. Therefore, quantization error should be considered in the active suspension design process. In addition, for active suspension systems, a certain amount of energy will be generated during the working process of the controller. The time-triggered mechanism [17] can be utilized to save energy and reduce the actuator action frequency while extending the life of the actuator.

This study proposes an event-trigger-based quantitative feedback active suspension system with quantization error, both the static quantizer and dynamic quantizer are utilized, and the sliding mode controller is also adopted. The objective of the proposed controller is to reduce the influence of accumulated sensor signal errors, enhance the actuator efficiency, and improve active suspension system properties. Compared with the recent active suspension control systems, the main contributions of this study are as follows:

(1) The connection channels between the suspension model and the controller are considered, and robust quantization control theory is designed for the active suspension system to reduce the signal transmission during data acquisition and transcoding

(2) The event trigger mechanism is introduced in the controller design process to reduce the operation frequency of the active suspension actuator
The rest of the paper is organized as follows. The 7-DOF suspension model with actuator dynamics is presented in the upcoming section. The procedure for the design of the quantized feedback controller and event trigger mechanism is illustrated next. Simulation and discussion of the results are summarized later, and concluding remarks are given in the last section.

\section{Vehicle Suspension Model}

A full vehicle suspension model with heave, pitch, and roll motions of the sprung mass and four unsprung mass vertical motions is utilized to describe the dynamics of the vehicle., as shown in Figure 1. The terms $m_{s}$ and $m_{u i}$ represent the sprung and unsprung masses, respectively. $\theta$ and $\varphi$ are the pitch and roll angle of the vehicle sprung mass. The linear damping and spring coefficients are denoted as $c_{i}$ and $k_{i}$, respectively, and $k_{\mathrm{ti}}$ represents the tire stiffness. The external road disturbance is denoted as $z_{r i}$. The suspension deflection can be expressed as

$$
z_{\text {sui }}=z_{s i}-z_{u i}, \quad(i=1,2,3,4)
$$

Then, the spring and damping forces can be given by

$$
\begin{aligned}
& F_{s i}=k_{i} z_{\text {sui }}+k_{\text {ni }} z_{\text {sui }}^{3}, \quad(i=1,2,3,4), \\
& F_{d i}=c_{i} \dot{z}_{\text {sui }}, \quad(i=1,2,3,4),
\end{aligned}
$$

where $k_{n i}$ is the nonlinear spring coefficient. $z_{s i}$ and $z_{u i}$ are the sprung and unsprung mass displacements of each corner, respectively. Assuming the pitch and roll angle of the sprung mass are small, the following approximation can be obtained:

$$
\begin{aligned}
& z_{s 1}=z_{s}+a \sin (\theta)+c \sin \varphi, \\
& z_{s 2}=z_{s}+a \sin (\theta)-d \sin \varphi, \\
& z_{s 3}=z_{s}-b \sin (\theta)+c \sin \varphi, \\
& z_{s 4}=z_{s}-b \sin (\theta)-d \sin \varphi,
\end{aligned}
$$

where $a, b, c$, and $d$ are the distances of the suspensions to the center of the vehicle. Then, the vehicle suspension model with sprung and unsprung mass dynamics can be given as

$$
\begin{aligned}
& m_{s} \ddot{z}_{s}+\sum\left(F_{s i}+F_{d i}-F_{a i}+F_{\mathrm{ati} 1}+F_{\mathrm{ati} 2}+F_{\mathrm{ati} 3}+F_{\mathrm{ati} 4}\right)=0, \\
& I_{y} \ddot{\theta}+a\left(F_{s 1}+F_{d 1}+F_{s 2}+F_{d 2}+F_{\mathrm{ati1}}+F_{\mathrm{ati} 2}\right)-b\left(F_{s 3}+F_{d 3}+F_{s 4}+F_{d 4}+F_{\mathrm{ati} 3}+F_{\mathrm{ati} 4}\right)-F_{\theta}=0, \\
& I_{x} \ddot{\varphi}-d\left(F_{s 2}+F_{d 2}+F_{\mathrm{ati} 2}+F_{s 4}+F_{d 4}+F_{\mathrm{ati} 4}\right)+c\left(F_{s 1}+F_{d 1}+F_{\mathrm{ati} 1}+F_{s 3}+F_{d 3}+F_{\mathrm{ati} 3}\right)-F_{\varphi}=0, \\
& m_{u i} \ddot{z}_{u i}-F_{s i}-F_{d i}+F_{t i}-F_{a i}=0, \quad(i=1,2,3,4),
\end{aligned}
$$

where $I_{x}$ and $I_{y}$ are the roll and pitch moment inertia of the sprung mass; $F_{\mathrm{ti}}=k_{\mathrm{ti}}\left(z_{u i}-z_{r i}\right)$ is the dynamic tire load. The antiroll bar forces are 


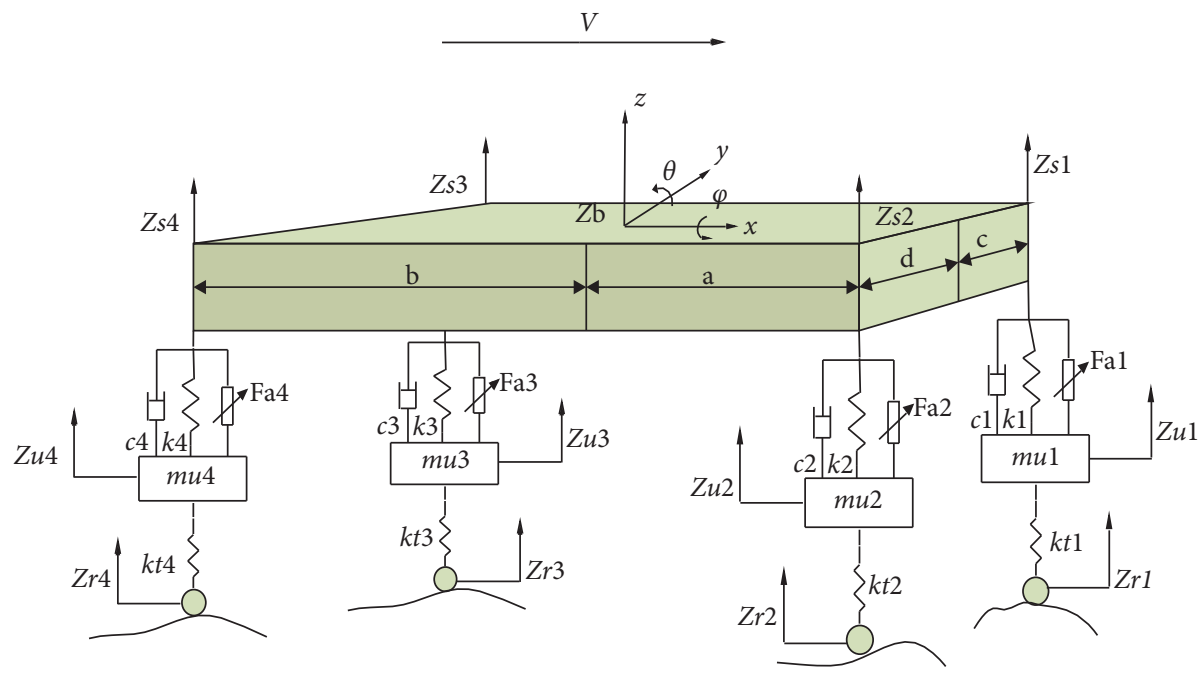

Figure 1: Active suspension model.

$$
\begin{aligned}
& F_{\mathrm{ati1}}=\frac{k_{a f}}{c+d}\left(\varphi-\frac{z_{u 2}-z_{u 1}}{c+d}\right), \\
& F_{\mathrm{ati} 2}=-\frac{k_{a f}}{c+d}\left(\varphi-\frac{z_{u 2}-z_{u 1}}{c+d}\right), \\
& F_{\mathrm{ati} 3}=-\frac{k_{r f}}{c+d}\left(\varphi-\frac{z_{u 3}-z_{u 4}}{c+d}\right), \\
& F_{\mathrm{ati} 4}=\frac{k_{r f}}{c+d}\left(\varphi-\frac{z_{u 3}-z_{u 4}}{c+d}\right),
\end{aligned}
$$

where $k_{a f}$ and $k_{\mathrm{r} f}$ are the antiroll bar stiffness.

Active force $F_{a i}$ is generated by the actuator located between the sprung and unsprung masses. The control input of the vertical, pitch, and roll motions can be expressed as

$$
\begin{aligned}
& F_{z}=F_{a 1}+F_{a 2}+F_{a 3}+F_{a 4}, \\
& F_{\varphi}=c\left(F_{a 1}+F_{a 3}\right)-d\left(F_{a 2}+F_{a 4}\right), \\
& F_{\theta}=a\left(F_{a 1}+F_{a 2}\right)-b\left(F_{a 3}+F_{a 4}\right) .
\end{aligned}
$$

The electrohydraulic actuator model can be represented as

$$
\dot{F}_{a i}=-\frac{C_{d}}{A_{p}} F_{a i}-\alpha A_{p}^{2} \dot{z}_{\text {sui }}+\lambda U_{i} \sqrt{P_{s}-\operatorname{sign}\left(U_{i}\right) \frac{F_{a i}}{A_{p}}},
$$

where $i=1,2,3,4, C_{d}$ is the discharge coefficient, $A_{p}$ is the actuator piston area, $P_{s}$ is the hydraulic supply pressure, $\alpha$ is a function of the bulk modulus of the fluid, $w$ is the spool valve area gradient, $\lambda=A_{p} C_{d} w \alpha / \sqrt{\rho}, \rho$ is the hydraulic fluid density, and $U_{i}$ is the spool valve input that can be the controlled voltage or current.

\section{Controller Design}

A quantize-event-trigger-based sliding mode controller is designed in this section for the active suspension system, and the control diagram of the proposed controller is shown in Figure 2 .

3.1. Robust Quantization Feedback Controller. Robust quantization control theory contains control problems based on the quantizer, in which the dynamic quantizer expands the quantization range by adjusting the quantization level to achieve asymptotic stability of the system. This section mainly focuses on the connection channels between the suspension model and the controller. Both static quantizer and dynamic quantizer are utilized, and a sliding mode controller is also adopted. The main feature of the dynamic quantizer is that it can increase the quantization range of the quantizer by adjusting the quantization level. It can also reduce the size of the limit cycle when the system is in a steady state and achieve asymptotic stability. Compared with the static quantizer, its control effect is better, but the structure is more complicated. For practical consideration, we utilize a static quantizer on the uplink channel and a dynamic quantizer on the downlink channel. The quantization system is shown in Figure 3.

Assuming the suspension system considered in this paper in bounded-state stable, the road profile and its derivate are totally bounded. Selecting the state variables as $\left[\begin{array}{llllll}x_{1} & x_{2} & x_{3} & x_{4} & x_{5} & x_{6}\end{array}\right]^{T}=\left[\begin{array}{llllll}z_{s} & \dot{z}_{s} & \theta & \dot{\theta} & \varphi & \dot{\varphi}\end{array}\right]^{T}$, the suspension system model including heave, pitch, and roll motions can be expressed as 


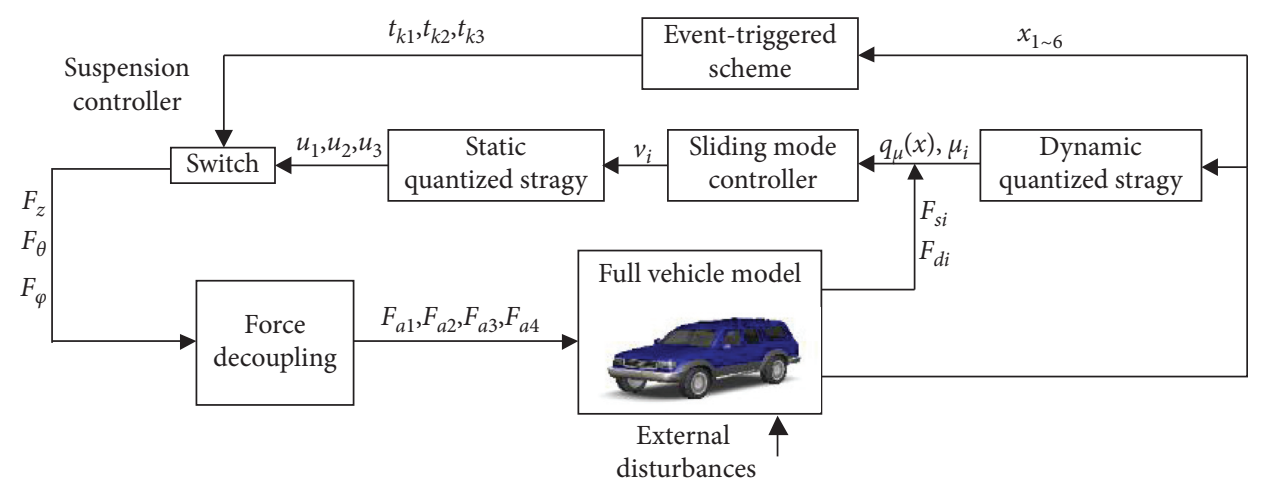

FIGURE 2: Diagram block of the proposed controller.

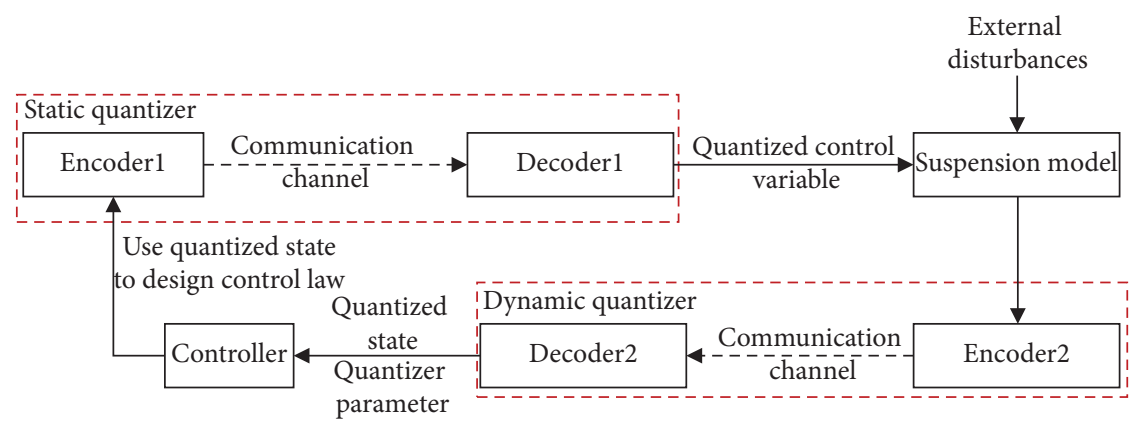

FIgURE 3: Flow chart of the quantization system.

$$
\begin{aligned}
& \dot{x}_{1}=x_{2}, \\
& \dot{x}_{2}=f_{z}+G_{z} F_{z}, \\
& f_{z}=-\frac{1}{m_{s}} \sum\left(F_{s i}+F_{d i}\right), \quad G_{z}=\frac{1}{m_{s}}, \\
& \dot{x}_{3}=x_{4}, \\
& \dot{x}_{4}=f_{\theta}+G_{\theta} F_{\theta}, \\
& f_{\theta}=-\frac{1}{I_{y}}\left[a\left(F_{s 1}+F_{d 1}+F_{s 2}+F_{d 2}\right)-b\left(F_{s 3}+F_{d 3}+F_{s 4}+F_{d 4}\right)\right], \quad G_{\theta}=\frac{1}{I_{y}}, \\
& \dot{x}_{5}=x_{6}, \\
& \dot{x}_{6}=f_{\varphi}+G_{\varphi} F_{\varphi}, \\
& f_{\varphi}=-\frac{1}{I_{x}}\left[c\left(F_{s 1}+F_{d 1}+F_{s 3}+F_{d 3}\right)-d\left(F_{s 2}+F_{d 2}+F_{s 4}+F_{d 4}\right)\right], \quad G_{\varphi}=\frac{1}{I_{x}} .
\end{aligned}
$$
[18]

Then, the abovementioned functions can be written as

$$
\mathbf{x}(t)=A_{0} \dot{x}(t)+B_{j}\left[\mathbf{U}_{\text {force }}+\mathbf{d}_{j}(t)\right],
$$

where $\mathbf{x}(t)=\left[x_{i}(t) ; x_{i+1}(t)\right] ; \quad i=1,3,5, \quad j=1,2,3$, and $\mathbf{U}_{\text {force }}=\left[F_{z} ; F_{\theta} ; F_{\varphi}\right]$ represent the vertical, pitch, and roll force generated by the active suspension, respectively.
$A_{0}=\left[\begin{array}{llll}0 & 1 ; 0 & 0\end{array}\right] ; B_{1}=\left[0 ; G_{z}\right], B_{2}=\left[0 ; G_{\theta}\right], B_{3}=\left[0 ; G_{\varphi}\right]$; and $\quad d_{1}(t)=\left[0 ;\left(f_{z} / G_{z}\right)\right], \quad d_{2}(t)=\left[0 ;\left(f_{\theta} / G_{\theta}\right)\right], \quad$ and $d_{3}(t)=\left[0 ;\left(f_{\varphi} / G_{\varphi}\right)\right]$.

Assumption 1. $\mathbf{d}_{j}(t)$ satisfies the inequality $\left|\mathbf{d}_{j}(t)\right| \leq d_{1}+d_{2}|\mathbf{x}(t)|+d_{3}\left|\mathbf{U}_{\text {force }}\right| \leq d_{4}$, where $\quad d_{1} \geq 0, d_{2}$ $\geq 0, d_{4}>0,0 \leq d_{3} \leq 1$, and $d_{1}, d_{2}, d_{3}, d_{4}$ are constants. 
As shown in Figure 3, a static quantizer $q_{\mu_{s}}(\mathbf{v})$ is used in the uplink channel, where $\mathbf{v}$ is the control law designed by the quantized state, $\mu_{s}$ is a fixed number; the down channel utilized a dynamic quantizer $q_{\mu}(\mathbf{x})$ to transfer system quantized states, and $\mu$ is the quantization parameter adjusted statically. Defining a function that takes the nearest integer, the uniform quantizer with the quantization level parameter $\mu$ is

$$
q_{\mu}(\mathbf{x}) \stackrel{\text { def }}{=} \mu_{j} \text { round }\left(\frac{\mathbf{x}}{\mu_{j}}\right), \quad \mu_{j}>0 ; j=1,2,3 .
$$

The uniform quantization error is

$$
\mathbf{e}_{\mu} \stackrel{\text { def }}{=} q_{\mu}(\mathbf{x})-\mathbf{x} .
$$

Then,

$$
\mathbf{e}_{\mu} \stackrel{\text { def }}{=}\left|q_{\mu}(\mathbf{x})-\mathbf{x}\right| \leq \Gamma \mu_{j},
$$

where $\Gamma=\sqrt{p} / 2, p$ is the dimension of the vector $\mathbf{x}$.

For the dynamic quantizer, a special quantization level parameter $\mu_{j}=0$ is given when the system runs to the sliding surface.

$$
q_{\mu}(\mathbf{x}) \stackrel{\text { def }}{=} 0, \quad \mu_{j}=0
$$

The linear sliding surface is defined as follows:

$$
s(\mathbf{x}(t))=C \mathbf{x}(t)=0,
$$

where $C$ is a given vector to ensure that the system has stable eigenvalues and $C B_{j} \neq 0$. The object of sliding mode control is to force the system state trajectory to reach the sliding surface and maintain the sliding mode in the presence of uncertainties and disturbances.

Lemma 1 (see [19]). There is a fixed constant $\tau>0$, and we assume $\mu>0$ which satisfies

$$
\mu \leq \frac{|C \mathbf{x}|}{(\tau+1)|C| \Gamma} .
$$

Then, the following equation can be obtained:

$$
\left|C \mathbf{e}_{\mu}\right| \leq|C| \Gamma \mu \leq \frac{1}{\tau}\left|C q_{\mu}(\mathbf{x})\right| .
$$

By considering the uplink and down channel quantize, the following control law can be designed [19]:

$$
\begin{aligned}
\mathbf{v}= & -\frac{\tau+1}{\tau-\chi}\left(C B_{j}\right)^{-1}\left[\left|C A q_{\mu}(\mathbf{x}(t))\right|+|C A| \Gamma \mu_{j}+\left|C B_{j}\right| \Gamma_{1} \mu_{s}+\left|C B_{j}\right|\left(d_{1}+d_{2}\left|q_{\mu}(\mathbf{x}(t))\right|+d_{2} \Gamma \mu_{j}+d_{3} \Gamma_{1} \mu_{s}\right)\right] \operatorname{sgn}\left[C q_{\mu}(\mathbf{x}(t))\right] \\
& -\frac{\tau \rho}{\tau-\chi}\left(C B_{j}\right)^{-1} \operatorname{sgn}\left[C q_{\mu}(\mathbf{x}(t))\right],
\end{aligned}
$$

$\mathbf{U}_{\text {force }}=q_{\mu_{s}}(\mathbf{v})$,

where $\chi=1+(\tau+1) d_{3}, \tau>\left(\left(1+d_{3}\right) /\left(1-d_{3}\right)\right), \rho>0$. The control law can keep the system states reach and maintain on the sliding surface $s(\mathbf{x}(t))=0$.

A simple and effective design of the adjustment law is as follows [20]:

$$
\begin{cases}\mu_{j}=\frac{\lfloor|C \mathbf{x}(t)|\rfloor}{(\tau+1)|C| \Gamma}, & |C \mathbf{x}(t)| \geq 1, \\ \mu_{j}=\frac{\alpha^{\lambda}}{(\tau+1)|C| \Gamma}, & 0<|C \mathbf{x}(t)|<1, \\ \mu_{j}=0, & |C \mathbf{x}(t)|=0,\end{cases}
$$

where $\alpha \in(0,1), \alpha^{\lambda}<|C \mathbf{x}|<\alpha^{\lambda-1}$.

3.2. Event Trigger Mechanism. In recent years, event triggers have been used to reduce computational costs and energy consumption, resulting in a wide range of applications in control systems $[21,22]$. The event trigger mechanism is based on the physical quantity signal of the sensor; when the semaphore reaches a certain condition, sampling is performed. In this way, the average sampling period is longer than the fixed sampling period, so that the sampling frequency of the system can be effectively reduced. As the nest sample instant is dependent on the previous sampling information, the control law is held constant between sampling instants. Defining the event trigger instant as $t_{k}$, the system maintains the control output at time $t_{k}$ in the time interval $\left[t_{k}, t_{k+1}\right]$. The discretization state error of the system is

$$
\mathbf{e}(t)=\mathbf{x}(t))-\mathbf{x}\left(t_{k}\right)
$$

The sampling is not periodic; hence, $t_{k+1}-t_{k}$ is not constant. For computational purposes, the deviation from the system state to the desired state is given by 


$$
\mathbf{e}_{T}=\mathbf{x}-\mathbf{x}_{d}
$$

where $\mathbf{x}_{d}$ is the set reference. As the error variable is required to vanish or settle in close vicinity of zero, the reference state is set as $\mathbf{x}_{d}=0$.

The triggering instant $t_{k}$ is completely characterized by a triggering rule, so a triggering rule is introduced [23].

$$
\kappa=\left\|\zeta \mathbf{e}_{T}+\xi \dot{e}_{T}^{2}\right\|-\psi\left(m_{1}+m_{2} \mathbf{e}^{-\varsigma t}\right),
$$

where $\zeta>0, \xi>0, \psi \in(0,1), \varsigma \in(0,1), m_{1} \geq 0, m_{2} \geq 0, m_{1}+$ $m_{2}>0$. The term $\left\|\zeta \mathbf{e}_{T}+\xi \dot{e}_{T}^{2}\right\|$ represents the overall error; the system is triggered when the error is small, guaranteeing the control output. When $\kappa \leq 0$, the system is triggered at $t_{k}$, and the control output is maintained until $t_{k+1}$. The following relation determines the triggering instants in an iterative manner:

$$
t_{k+1}=\inf \left\{t \in\left[t_{k}, \infty\right], \quad \kappa>0\right\} .
$$

The event trigger time is $T_{k}=t_{k+1}-t_{k}$. For $T_{k} \leq$ $t_{k+1}-t_{k}$, and lower bound on the event trigger time is ensured. $T_{k}$ is bounded by [23]

$$
T_{k} \geq \frac{1}{\|A\|} \ln \left(\frac{\|A\| \cdot\|\mathbf{e}\|_{\infty}}{\|A\| \Phi+\left\|B_{j}\right\|\left(q_{\mu_{s}}(\mathbf{v})+d_{4}\right)}+1\right)
$$

where $₫>0$.

Proof: Considering system (9),

$$
\dot{x}(t)=A \mathbf{x}(t)+B_{j} \mathbf{U}_{\text {force }}(t)+B_{j} \mathbf{d}_{j}(t)
$$

At time interval $\left[t_{k}, t_{k+1}\right]$, discretization state error rises from 0 to the final value at $T_{k}$ instant, so

$$
\begin{aligned}
\frac{\mathrm{d}}{\mathrm{d} t}\|\mathbf{e}(t)\| & \leq \frac{\mathrm{d}}{\mathrm{d} t}\|\overline{\mathbf{e}}(t)\| \leq \frac{\mathrm{d}}{\mathrm{d} t}\|\mathbf{x}(t)\| \\
& \leq\left\|A \mathbf{x}(t)+B_{j} \mathbf{U}_{\text {force }}(t)+B_{j} \mathbf{d}_{j}(t)\right\|,
\end{aligned}
$$

by inserting the control law (17) into abovementioned equation.

$$
\begin{aligned}
\frac{\mathrm{d}}{\mathrm{d} t}\|\mathbf{e}(t)\| & \leq\left\|A \mathbf{x}(t)+B_{j} q_{\mu_{s}}(\mathbf{v})+B_{j} \mathbf{d}_{j}(t)\right\| \\
& \leq\|A \mathbf{x}(t)\|+\left\|B_{j} q_{\mu_{s}}(\mathbf{v})\right\|+\left\|B_{j} \mathbf{d}_{j}(t)\right\| \\
& \leq\left\|A\left(\mathbf{e}(t)+\operatorname{txn}\left(t_{k}\right)\right)\right\|+\left\|B_{j} q_{\mu_{s}}(\mathbf{v})\right\|+\left\|B_{j} \mathbf{d}_{j}(t)\right\| \\
& \leq\|A \mathbf{e}(t)\|+\left\|A \mathbf{x}\left(t_{k}\right)\right\|+\left\|B_{j} q_{\mu_{s}}(\mathbf{v})\right\|+\left\|B_{j} \mathbf{d}_{j}(t)\right\| \\
& \leq\|A\|\|\mathbf{e}(t)\|+\|A\| \Phi+\left\|B_{j}\right\|\left(q_{\mu s}(\mathbf{v})+d_{4}\right) .
\end{aligned}
$$

$\forall t \in\left[t_{k}, t_{k+1}\right]$, with the initial condition $\mathbf{e}(t)=0$, and the solution of inequality (27) can be understood by utilizing the comparison lemma [24] and is given as

$$
\|\mathbf{e}(t)\| \leq \frac{\|A\| \omega+\left\|B_{j}\right\|\left(q_{\mu s}(\mathbf{v})+d_{4}\right)}{\|A\|}\left(\mathbf{e}^{\|A\|\left(t-t_{k}\right)}-1\right) .
$$

Similarly, for triggering time instant $t_{k+1}$,

$$
\left\|\mathbf{e}_{\infty}=\right\| \mathbf{e}\left(t_{k+1}\right) \| \leq \frac{\|A\| \bowtie+\left\|B_{j}\right\|\left(q_{\mu_{s}}(\mathbf{v})+d_{4}\right)}{\|A\|}\left(\mathbf{e}^{\|A\| T_{k}}-1\right)
$$

Discretized state error after quantization is

$$
\mathbf{e}_{q}(t)=q_{\mu}(\mathbf{x}(t))-q_{\mu}\left(\mathbf{x}\left(t_{k}\right)\right) .
$$

Event is triggered at discrete moments $\forall t \in\left[t_{k}, t_{k+1}\right]$, when $s(t)>0$ or $s(t)<0, s(t)=s\left(t_{k}\right)$. So, the following equation is satisfied:

$$
\operatorname{sgn}\left(C q_{\mu}(\mathbf{x}(t))\right)=\operatorname{sgn}\left(C q_{\mu}\left(\mathbf{x}\left(t_{k}\right)\right)\right), \quad t \in\left[t_{k}, t_{k+1}\right] .
$$

Therefore, the triggered control law applied to the system

and then, inequality (24) is satisfied.

$$
\begin{aligned}
\mathbf{F}_{a i}= & \mathbf{U}_{\text {force }}=-\frac{\tau+1}{\tau-\chi}\left(C B_{j}\right)^{-1}\left[\left|C A q_{\mu}\left(\mathbf{x}\left(t_{k}\right)\right)\right|+|C A| \Gamma \mu_{j}+\left|C B_{j}\right| \Gamma_{1} \mu_{s}+\left|C B_{j}\right|\left(d_{1}+d_{2}\left|q_{\mu}\left(\mathbf{x}\left(t_{k}\right)\right)\right|+d_{2} \Gamma \mu_{j}+d_{3} \Gamma_{1} \mu_{s}\right)\right] \\
& \cdot \operatorname{sgn}\left[C q_{\mu}\left(\mathbf{x}\left(t_{k}\right)\right)\right]-\frac{\tau \rho}{\tau-\chi}\left(C B_{j}\right)^{-1} \operatorname{sgn}\left[C q_{\mu}\left(\mathbf{x}\left(t_{k}\right)\right)\right] .
\end{aligned}
$$


3.3. Stability Analysis. In order to illustrate the reliability of the proposed algorithm, the stability of the quantize-eventtrigger-based sliding mode controller needs to be considered. From (11) and (18), $\mathbf{e}_{\mu_{s}}=q_{\mu_{s}}(\mathbf{v})-\mathbf{v}=\mathbf{U}_{\text {force }}-\mathbf{v}$; then, $\mathbf{U}_{\text {force }}=q_{\mu_{s}}(\mathbf{v})=\mathbf{v}+\mathbf{e}_{\mu_{s}}$. Combining with $\mathbf{x}(t)=q_{\mu}(\mathbf{x}(t))$ $-\mathbf{e}_{\mu}$, the derivative of the sliding surface is written as

$$
\begin{aligned}
\dot{\boldsymbol{s}}(\mathbf{x}(t)) & =C \dot{x}(t)=C\left[A\left(q_{\mu}(\mathbf{x}(t))-e_{\mu}\right)+B_{j}\left(\mathbf{v}+\mathbf{e}_{\mu_{s}}+\mathbf{d}_{j}\right)\right] \\
& =C\left[A q_{\mu}(\mathbf{x}(t))-A \mathbf{e}_{\mu}+B_{j} \mathbf{v}+B_{j} \mathbf{e}_{\mu_{s}}+B_{j} \mathbf{d}_{j}\right] .
\end{aligned}
$$

From inequalities $\mathbf{e}_{\mu}=q_{\mu}(\mathbf{x})-\mathbf{x} \mid \leq \Gamma \mu_{j}$ and $C \mathbf{e}_{\mu}|\leq| C \mid$ $\Gamma \mu_{j} \leq|1 / \tau| C q_{\mu}(\mathbf{x}) \mid$,

$$
\begin{aligned}
s(\mathbf{x}(t)) \dot{s}(\mathbf{x}(t))= & C q_{\mu}(\mathbf{x}(t))\left\{C\left[A q_{\mu}(\mathbf{x}(t))-A \mathbf{e}_{\mu}+B_{j} \mathbf{v}+B_{j} \mathbf{e}_{\mu_{s}}+B_{j} \mathbf{d}_{j}\right]\right\} \\
& -C \mathbf{e}_{\mu}\left\{C\left[A q_{\mu}(\mathbf{x}(t))-A \mathbf{e}_{\mu}+B_{j} \mathbf{v}+B_{j} \mathbf{e}_{\mu_{s}}+B_{j} \mathbf{d}_{j}\right]\right\} \\
\leq & \frac{\tau+1}{\tau}\left|C q_{\mu}(\mathbf{x}(t))\right|\left|C A q_{\mu}(\mathbf{x}(t))\right|+\frac{\tau+1}{\tau}\left|C q_{\mu}(\mathbf{x}(t))\right||C A| \Gamma \mu_{j}+\frac{\tau+1}{\tau}\left|C q_{\mu}(\mathbf{x}(t))\right|\left|C B_{j}\right| \Gamma_{1} \mu_{s} \\
& +\frac{\tau+1}{\tau}\left|C q_{\mu}(\mathbf{x}(t))\right|\left|C B_{j}\right|\left|\mathbf{d}_{j}\right|+\frac{1}{\tau}\left|C q_{\mu}(\mathbf{x}(t)) \| C B_{j} \mathbf{v}\right|+C q_{\mu}(\mathbf{x}(t)) C B_{j} \mathbf{v} .
\end{aligned}
$$

If Assumption 1 is satisfied, equation (34) can be written

as

$$
\begin{aligned}
s(\mathbf{x}(t)) \dot{s}(\mathbf{x}(t)) \leq & \frac{\tau+1}{\tau}\left|C q_{\mu}(\mathbf{x}(t))\right|\left|C A q_{\mu}(\mathbf{x}(t))\right|+\frac{\tau+1}{\tau}\left|C q_{\mu}(\mathbf{x}(t))\right||C A| \Delta \mu_{j}+\frac{\tau+1}{\tau}\left|C q_{\mu}(\mathbf{x}(t))\right|\left|C B_{j}\right| \Gamma_{1} \mu_{s} \\
& +\frac{\tau+1}{\tau}\left|C q_{\mu}(\mathbf{x}(t))\right| C B_{j} \mid\left(d_{1}+d_{2}\left|q_{\mu}(\mathbf{x}(t))\right|+d_{2} \Gamma \mu_{j}+d_{3}\left|\mathbf{U}_{\text {force }}\right|\right) \\
& +\frac{1}{\tau}\left|C q_{\mu}(\mathbf{x}(t))\right|\left|C B_{j} v\right|+C q_{\mu}(\mathbf{x}(t)) C B_{j} v .
\end{aligned}
$$

As $\left|\mathbf{U}_{\text {force }}\right|=\left|q_{\mu_{1}}(\mathbf{v})\right|=\left|\mathbf{v}+\mathbf{e}_{\mu_{s}}\right| \leq|\mathbf{v}|+\Gamma_{1} \mu_{s}$ and $\chi=1+$ $(\tau+1) d_{3}$, equation (35) can be written as

$$
\begin{aligned}
s(\mathbf{x}) \dot{s}(\mathbf{x}) \leq & \frac{\tau+1}{\tau}\left|C q_{\mu}(\mathbf{x})\right|\left|C A q_{\mu}(\mathbf{x})\right|+\frac{\tau+1}{\tau}\left|C q_{\mu}(\mathbf{x})\right||C A| \Gamma \mu_{j}+\frac{\tau+1}{\tau}\left|C q_{\mu}(\mathbf{x})\right|\left|C B_{j}\right| \Gamma_{1} \mu_{s} \\
& +\frac{\tau+1}{\tau}\left|C q_{\mu}(\mathbf{x})\right|\left|C B_{j}\right|\left(d_{1}+d_{2}\left|q_{\mu}(\mathbf{x})\right|+d_{2} \Gamma \mu_{j}+d_{3} \Gamma_{1} \mu_{s}\right) \\
& +\frac{\chi-1}{\tau}\left|C q_{\mu}(\mathbf{x})\right|\left|C B_{j}\right||\mathbf{v}|+\frac{1}{\tau}\left|C q_{\mu}(\mathbf{x})\right|\left|C B_{j} \mathbf{v}\right|+C q_{\mu}(\mathbf{x}) C B_{j} \mathbf{v}, \\
= & \frac{\tau+1}{\tau}\left|C q_{\mu}(\mathbf{x})\right|\left|C A q_{\mu}(\mathbf{x})\right|+\frac{\tau+1}{\tau}\left|C q_{\mu}(\mathbf{x})\right||C A| \Gamma \mu_{j}+\frac{\tau+1}{\tau}\left|C q_{\mu}(\mathbf{x})\right|\left|C B_{j}\right| \Gamma_{1} \mu_{s} \\
& +\frac{\tau+1}{\tau}\left|C q_{\mu}(\mathbf{x}) \| C B_{j}\right|\left(d_{1}+d_{2}\left|q_{\mu}(\mathbf{x})\right|+d_{2} \Gamma \mu_{j}+d_{3} \Gamma_{1} \mu_{s}\right) \\
& +\frac{\chi}{\tau}\left|C q_{\mu}(\mathbf{x}) \| C B_{j}\right||\mathbf{v}|+\frac{\tau-\chi}{\tau} C q_{\mu}(\mathbf{x}) C B_{j} \mathbf{v}+\frac{\chi}{\tau} C q_{\mu}(\mathbf{x}) C B_{j} \mathbf{v} .
\end{aligned}
$$


For $\tau>\left(\left(1+d_{3}\right) /\left(1-d_{3}\right)\right), \quad d_{3}<((\tau-1) /(\tau+1)), \quad \chi=$ $1+(\tau+1) d_{3}<\tau$. By substituting the control law (17), the following inequality can be obtained:

$$
s(\mathbf{x}(t)) \dot{\boldsymbol{s}}(\mathbf{x}(t)) \leq-\rho\left|C q_{\mu}(\mathbf{x}(t))\right|-\frac{\tau+1}{\tau}\left|C q_{\mu}(\mathbf{x}(t))\right|\left(\left|C A \mathbf{e}_{q}\right|+\left|C B_{j}\right| d_{2}\left|\mathbf{e}_{q}\right|\right) .
$$

Defining $\sigma=\rho+((\tau+1) / \tau)\left(\left|C A \mathbf{e}_{q}\right|+\left|C B_{j}\right| d_{2}\left|\mathbf{e}_{q}\right|\right)$, (37) can be written as

$$
s(\mathbf{x}(t)) \dot{s}(\mathbf{x}(t)) \leq-\sigma\left|C q_{\mu}(\mathbf{x}(t))\right| .
$$

According to $\left|C \mathbf{e}_{\mu}\right| \leq|C| \Gamma \mu_{j} \leq(1 / \tau)\left|C q_{\mu}(\mathbf{x})\right|$ and triangle inequality, one can obtain

$$
\begin{aligned}
|C \mathbf{x}(t)| & =\left|C q_{\mu}(\mathbf{x}(t))-C \mathbf{e}_{\mu}\right| \leq\left|C q_{\mu}(\mathbf{x}(t))\right|+\left|C \mathbf{e}_{\mu}\right| \\
& \leq \frac{\tau+1}{\tau}\left|C q_{\mu}(\mathbf{x}(t))\right| \Rightarrow \frac{\tau}{\tau+1}|C \mathbf{x}(t)| \leq\left|C q_{\mu}(\mathbf{x}(t))\right| .
\end{aligned}
$$

Then, (38) it can be observed as

$$
\begin{aligned}
s(\mathbf{x}(t)) \dot{\boldsymbol{s}}(\mathbf{x}(t)) & \leq-\rho\left|C q_{\mu}(\mathbf{x}(t))\right| \leq-\frac{\tau}{\tau+1}|C \mathbf{x}(t)|, \\
& =-\frac{\tau \rho}{\tau+1}|s(\mathbf{x}(t))| .
\end{aligned}
$$

Hence, the state trajectories of the plant can be driven to the sliding surface, and the overall stability of the proposed suspension controller holds.

\section{Numerical Simulation and Discussion}

The effectiveness of the proposed controller is discussed in this section through the simulation results of the MATLAB/ Simulink. The model and controller parameters are listed in Tables 1 and 2, respectively.

When the vehicle is driven in a straight line, road roughness is an important factor that affects the riding comfort and handling stability of the vehicle. Pavement roughness is often expressed in terms of statistical characteristic parameters in the frequency domain, such as power spectral density or variance. However, the power spectral density expression can hardly meet the research needs of vehicle dynamics control, and it is necessary to simulate the road roughness in the time domain. The power spectral density of signal wheel road disturbance can be expressed as [25]

$$
G_{q}\left(n_{0}\right)=G_{q}\left(n_{0}\right)\left(\frac{n}{n_{0}}\right)^{-W}
$$

Road roughness $q(t)$ can be seen as the first-order response of unite white noise in the filtered white noise method, and through introducing a low cutoff frequency, the system frequency response is

$$
H_{q \sim \omega}(\omega)=\frac{2 \pi n_{0} \sqrt{G_{q}\left(n_{0}\right) v}}{j \omega+2 \pi n_{00} v} .
$$

The time-domain model of signal wheel excitation can be obtained by writing (42) into a differential form.

$$
\dot{q}(t)=2 \pi n_{0} \sqrt{G_{q}\left(n_{0}\right) v} w(t)-2 \pi n_{00} v q(t) .
$$

The definition of specific symbols can be seen in [25]. The four-wheel excitation model can be obtained by the time delay between front and rear axles and the coherence between left and right tracks [26]. Assuming that the vehicle is traveling on the B-level road with constant speed of $60 \mathrm{~km} / \mathrm{h}$, the time domain road profiles of four wheels are plotted in Figure 4.

The purpose of the active suspension controller is to improve the riding comfort while keeping the wheels in constant contact with the road surface. The riding comfort is evaluated by the movement of the sprung mass motion, and the handling stability is evaluated through the dynamic displacement of the tire. To verify the effectivness of the quantized feedback controller, different quantitative and trigger conditions are considered, and the road disturbance input is the B-level road.

Figure 5-Figure 7 reflect the control effect of the sprung mass motions with the quantized feedback controller. Sprung mass vertical acceleration, pitch acceleration, and roll acceleration with the combined quantizer, static quantizer, and uncontrolled condition are plotted. As can be seen from the figures, the passive system reported significantly more amplitude than the other two controlled systems. Partial enlarged detail of the figures indicates that compared with the static quantizer, a combined quantizer composed of the static and dynamic quantizer can achieve better performance.

Figure 8 represents the simulation contrast curves of the suspension deflection of the vehicle. It can be seen from Figure 8 that the proposed combined quantizer in this study outperforms the static quantizer and passive system in time domain. The time responses of the vehicle's tire deflection of four corners are set out in Figure 9. As shown in the figure, although the peak value of the controlled system is slightly greater than that of the passive system, the root mean square values are nearly consistent with the uncontrolled model. The partial enlarged details also show that the tire deflections with the static and combined quantizer are little changed. These results suggest that the quantized feedback controller is able to improve riding comfort effectively while maintaining the wheels in contact with the road surface. 
TABLE 1: Suspension model parameters.

\begin{tabular}{lc}
\hline Parameter & Value \\
\hline Sprung mass $m_{s}$ & $1592 \mathrm{~kg}$ \\
Unsprung mass $m_{u i}$ & $120 / 150 \mathrm{~kg}$ \\
Gravity to front axle distance $a$ & $1.18 \mathrm{~m}$ \\
Gravity to rear axle distance $b$ & $1.77 \mathrm{~m}$ \\
Center of gravity to the left side $c$ & $0.7875 \mathrm{~m}$ \\
Center of gravity to the right side $d$ & $0.7875 \mathrm{~m}$ \\
Pitch moment of inertia $I_{y}$ & $2488 \mathrm{~kg} \bullet \mathrm{m}^{2}$ \\
Roll moment of inertia $I_{x}$ & $614 \mathrm{~kg} \bullet \mathrm{m}^{2}$ \\
Spring stiffness $k_{i}$ & $146000 \mathrm{~N} / \mathrm{m}$ \\
Damping coefficient $b_{i}$ & $3000 \mathrm{Nm} / \mathrm{s}$ \\
Tire stiffness $k_{t i}$ & $230000 \mathrm{~N} / \mathrm{m}$ \\
Hydraulic supply pressure $P_{s}$ & $1.034 \times 10^{7} \mathrm{~N} / \mathrm{m}^{2}$ \\
Piston area $A_{p}$ & $3.35 \times 10^{-4}$ \\
Discharge coefficient $C_{d}$ & 0.61 \\
Spool valve area gradient $w$ & $1.436 \times 10^{-2} \mathrm{~m}$ \\
Oil density $\rho$ & $858 \mathrm{~kg} / \mathrm{m}^{3}$ \\
$\alpha$ & $1.19143 \times 10^{13}$ \\
\hline
\end{tabular}

TABle 2: Controller parameters.

\begin{tabular}{lc}
\hline Parameter & Value \\
\hline$d_{1}$ & 0.1 \\
$d_{2}$ & 0.1 \\
$d_{3}$ & 0.01 \\
$\alpha$ & 0.5 \\
$\rho$ & 4 \\
$\mu_{s}$ & 0.05 \\
$p_{1}$ & 0.8 \\
$p_{2}$ & 0.8 \\
$p_{3}$ & 0.05 \\
$p_{4}$ & $1 e-5$ \\
$p_{5}$ & 0 \\
$p_{6}$ & 0.97 \\
\hline
\end{tabular}

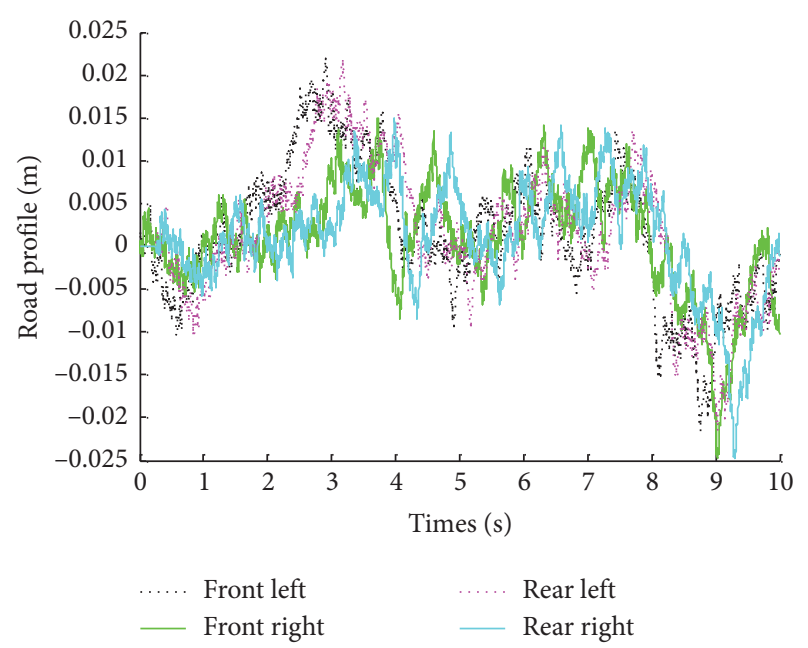

Figure 4: Four-wheel excitation input.

The results of the trigger signals are shown in Figure 10, and the quantization parameters are summarized in Figure 11. From the data in Figures 10 and 11, it is apparent that

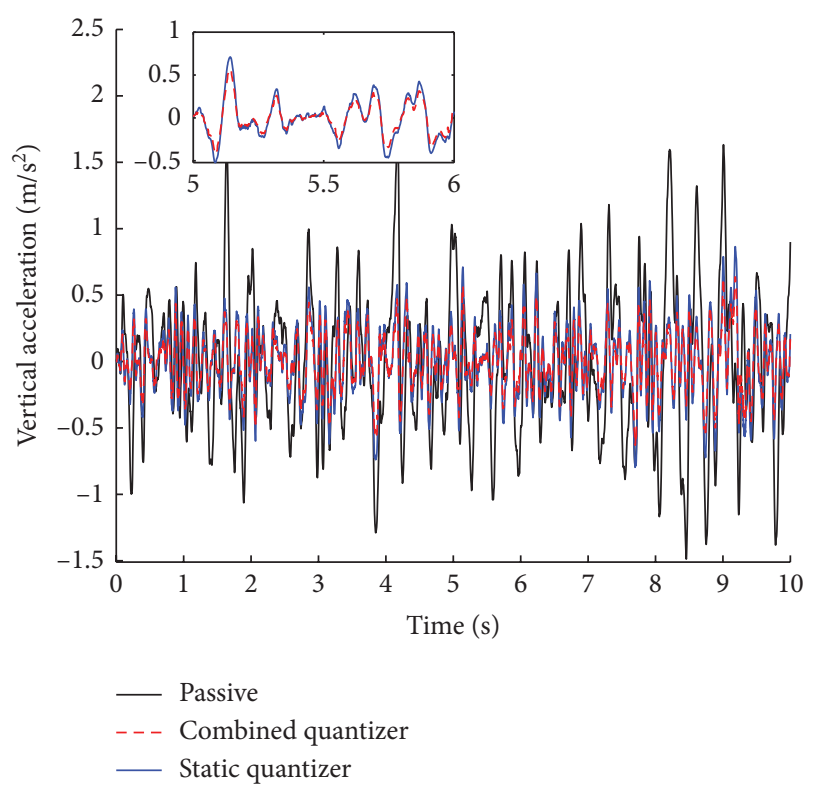

FIgURE 5: Sprung mass vertical acceleration.

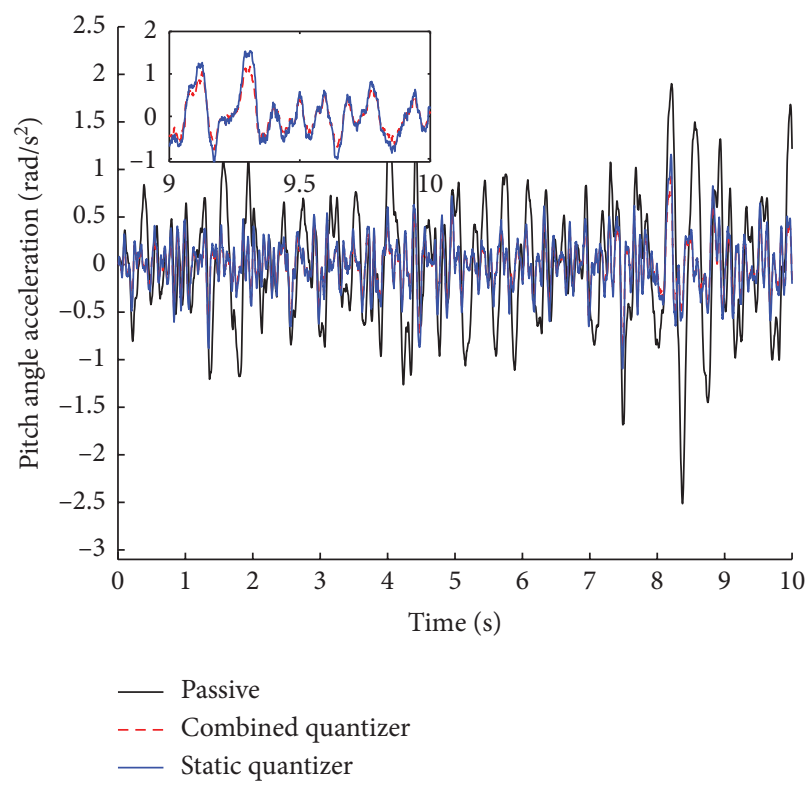

FIGURE 6: Sprung mass pitch acceleration.

the trigger moments are corresponding to the change of quantization parameters. Actuator force with the event trigger mechanism is presented in Figure 12. The figure illustrates that compared with the control law without the trigger mechanism, the output force frequency with the trigger mechanism is significantly reduced, which has a certain effect on reducing power consumption and reducing fatigue damage of the actuator. Therefore, it is necessary to introduce the trigger mechanism to the controller.

Table 3 compares the summary statistics for sprung mass accelerations, suspension deflection, and tire deflection with and without the trigger mechanism. Besides, the nonsingular fast terminal sliding mode (NFTSM) control method [27] is 


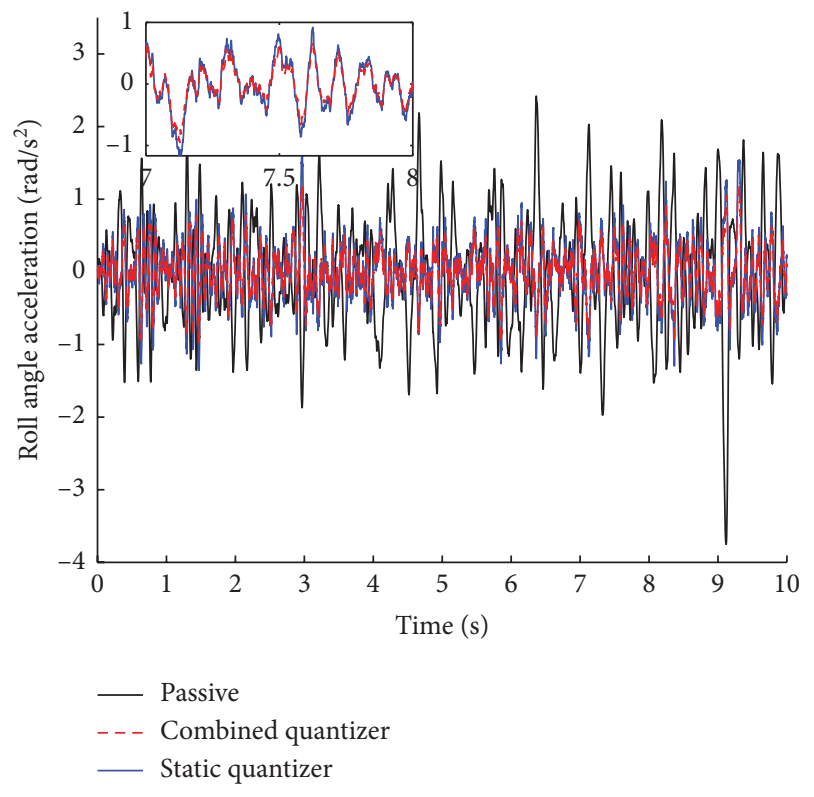

FIGURE 7: Sprung mass roll acceleration.

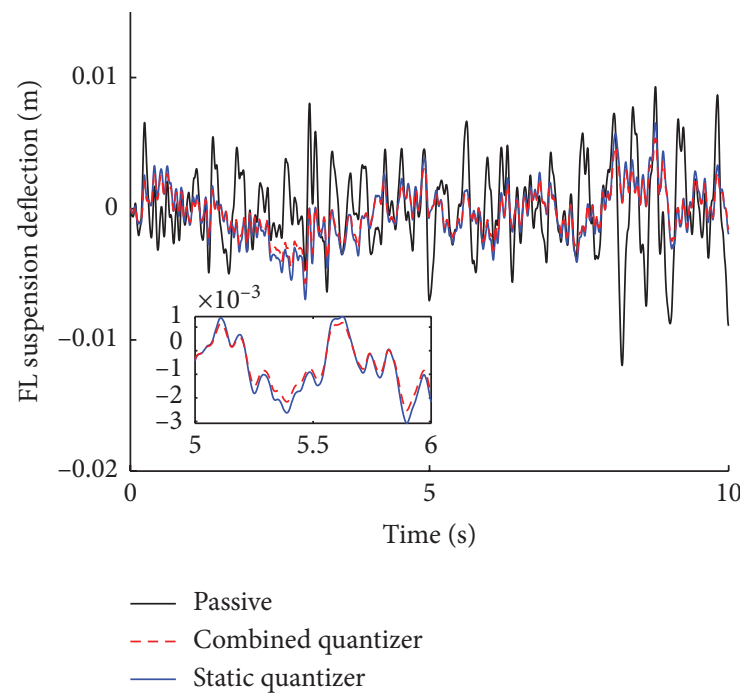

(a)

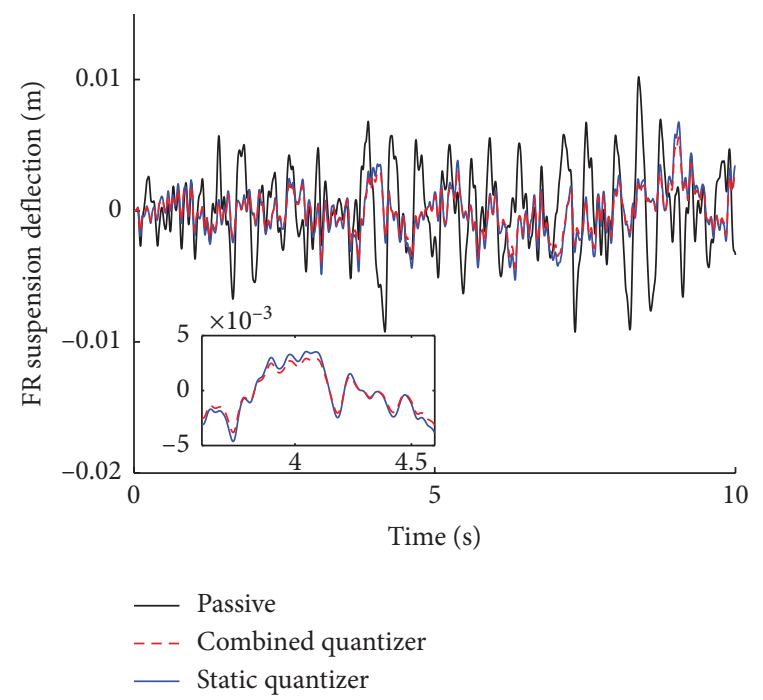

(b)

Figure 8: Continued. 

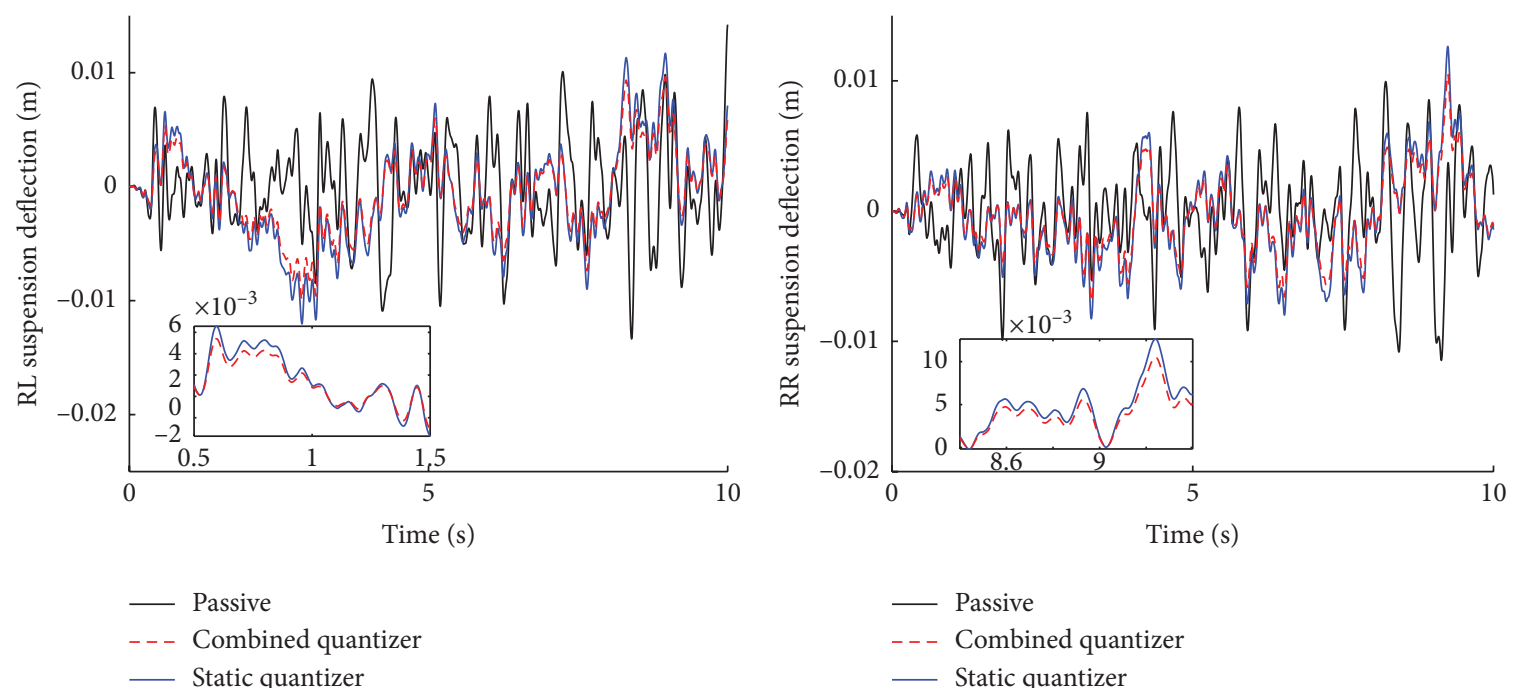

(c)

(d)

Figure 8: Suspension deflection.

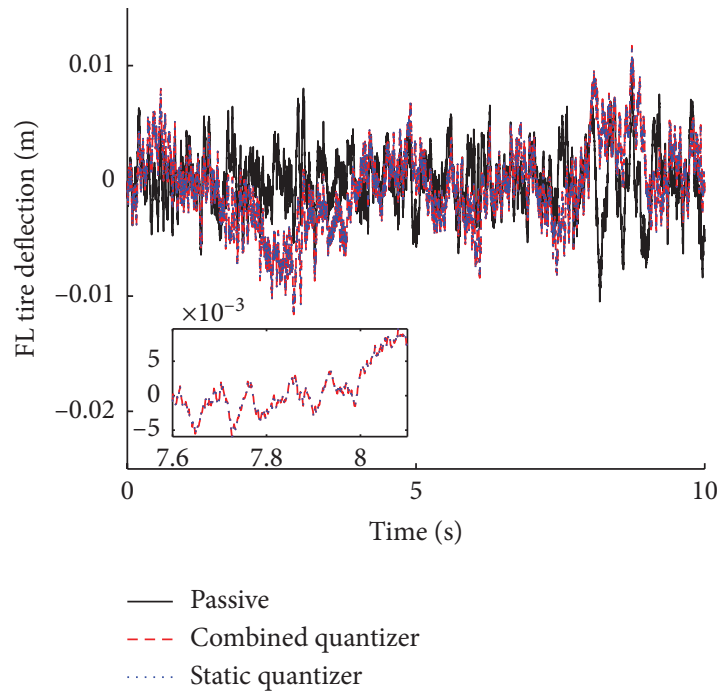

(a)

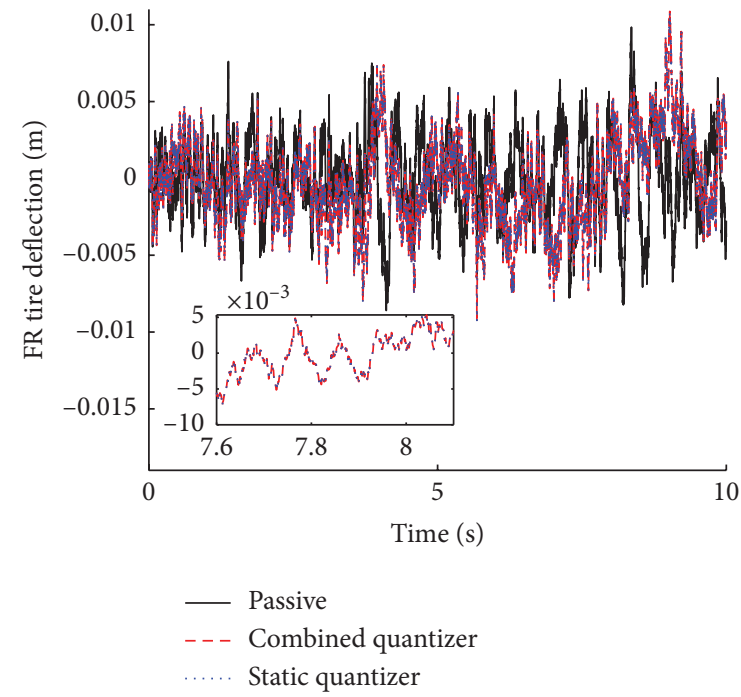

(b)

Figure 9: Continued. 

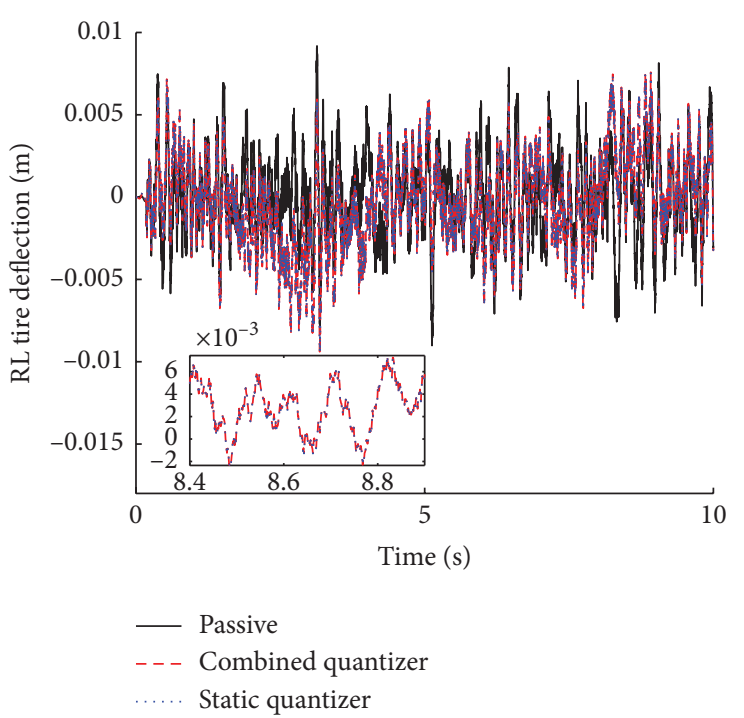

(c)

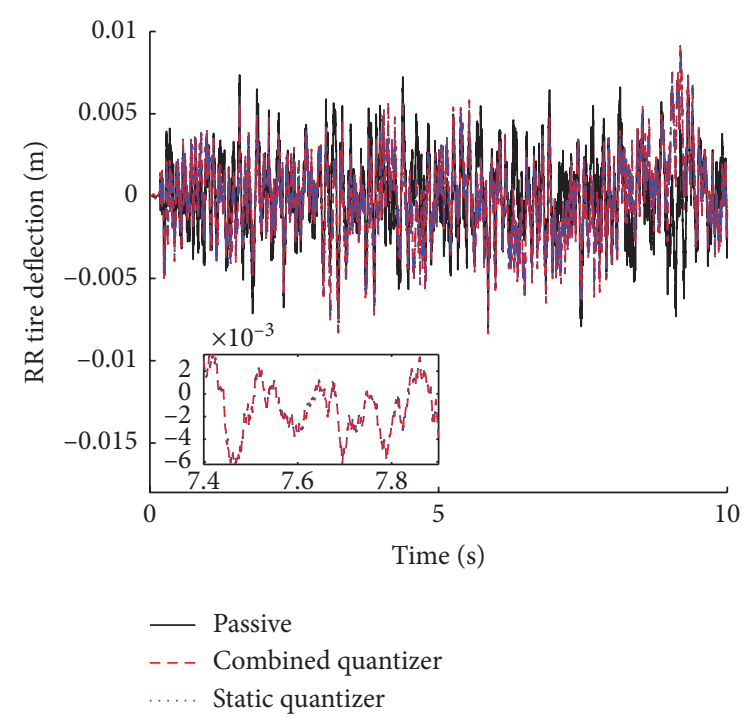

(d)

Figure 9: Tire deflection.

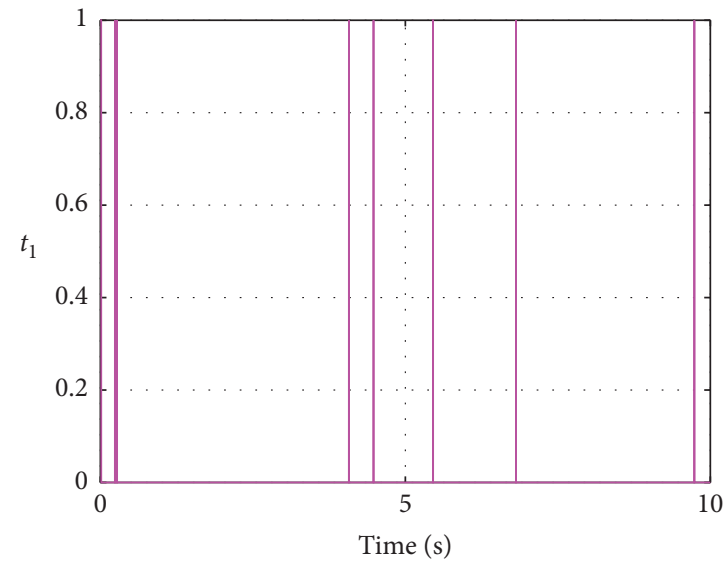

_ Trigger signal

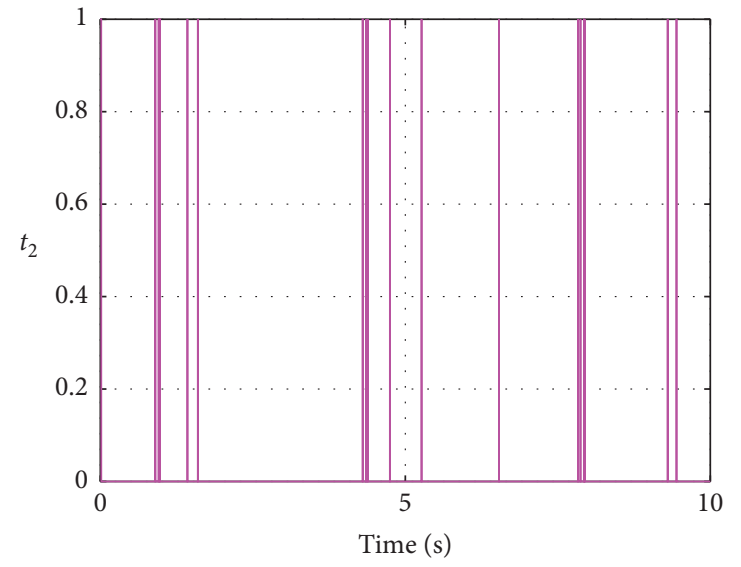

__ Trigger signal

(a)

(b)

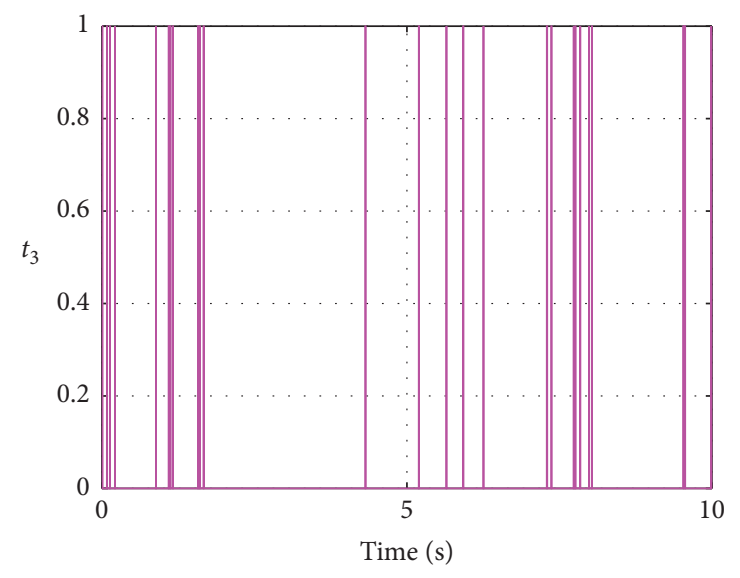

_ Trigger signal

(c)

FIgURE 10: Trigger signals. 


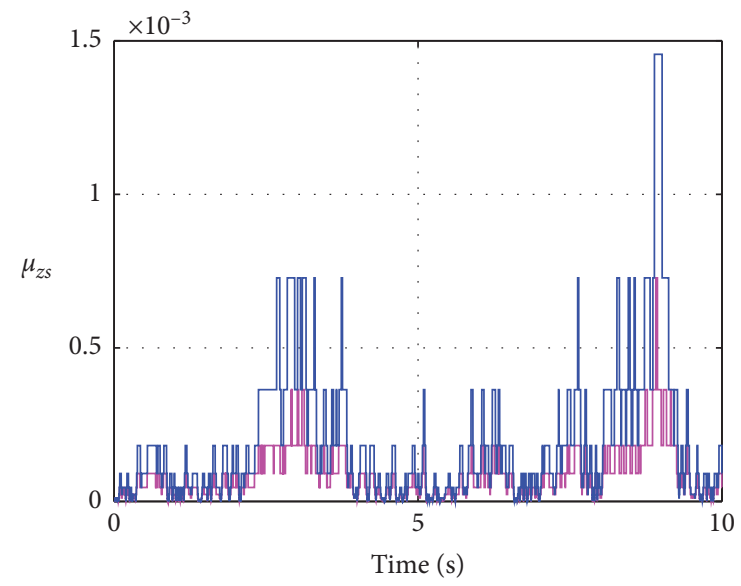

_ With event trigger Without event trigger

(a)

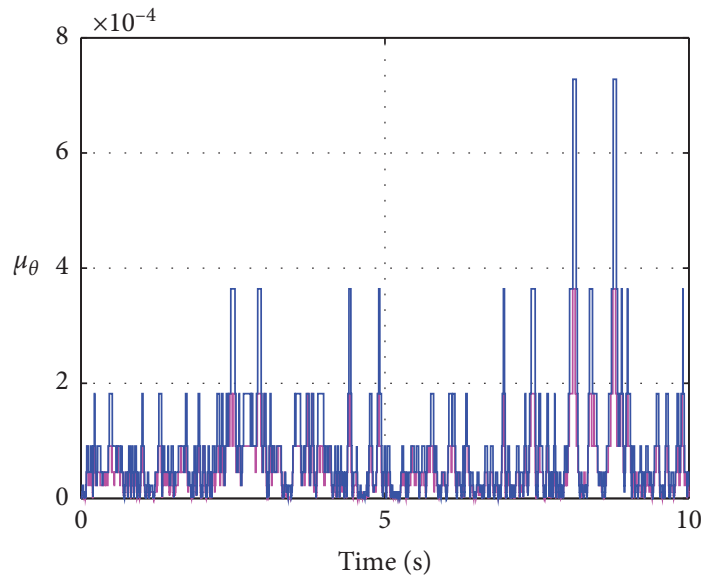

— With event trigger

_ Without event trigger

(b)

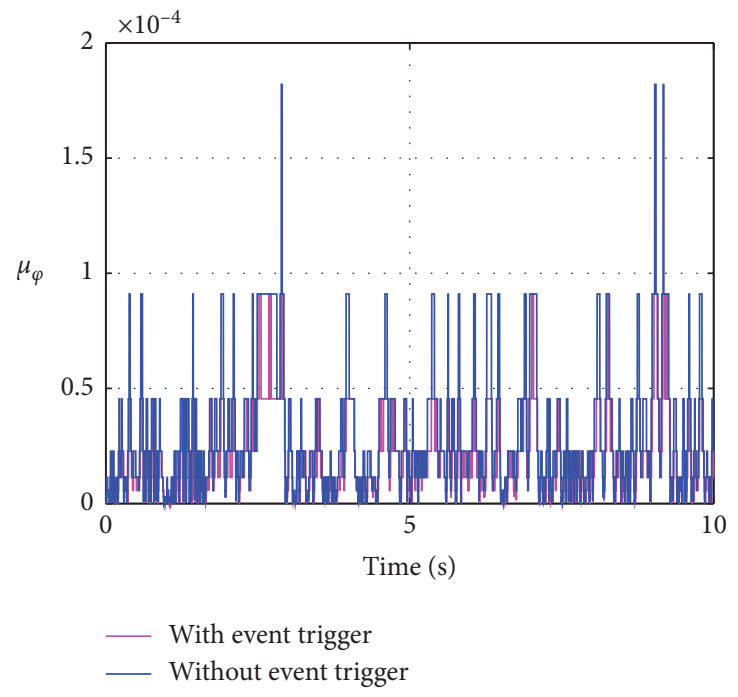

(c)

FIGURE 11: Quantization parameters.

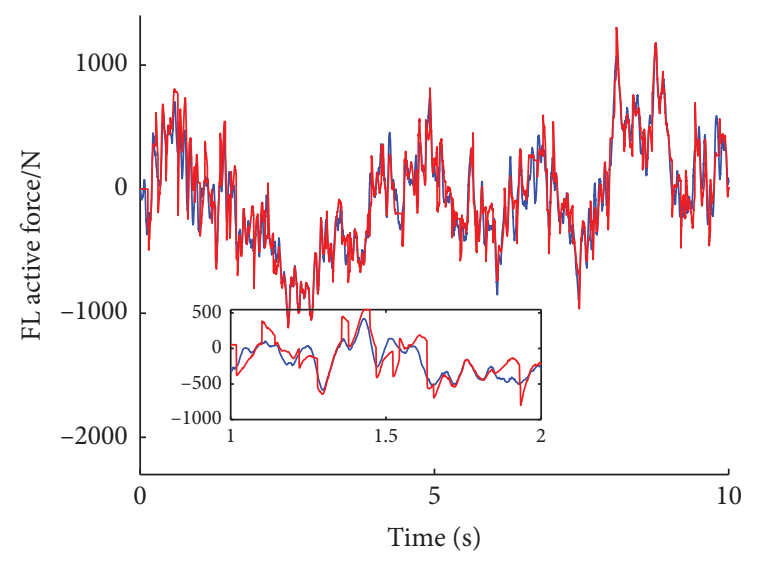

- Without event trigger

_ With event trigger

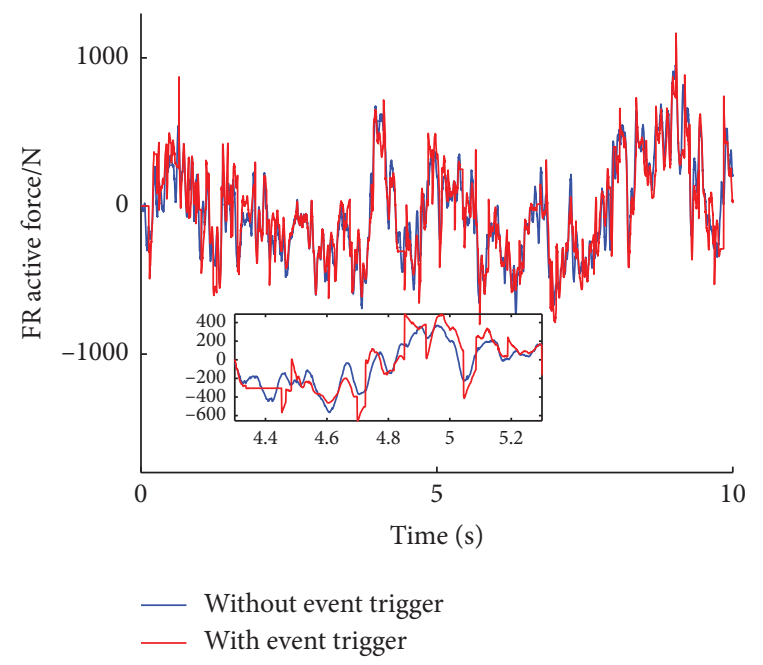

(b)

Figure 12: Continued. 


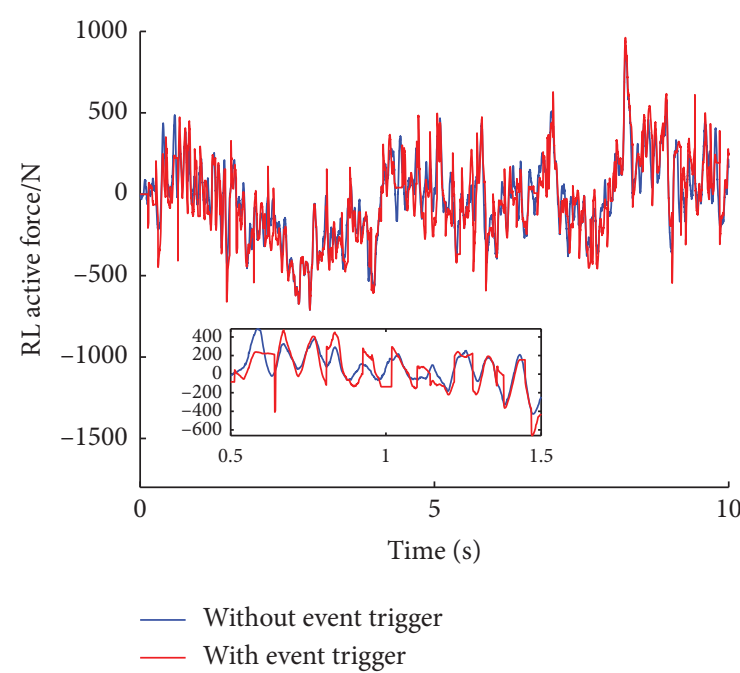

(c)

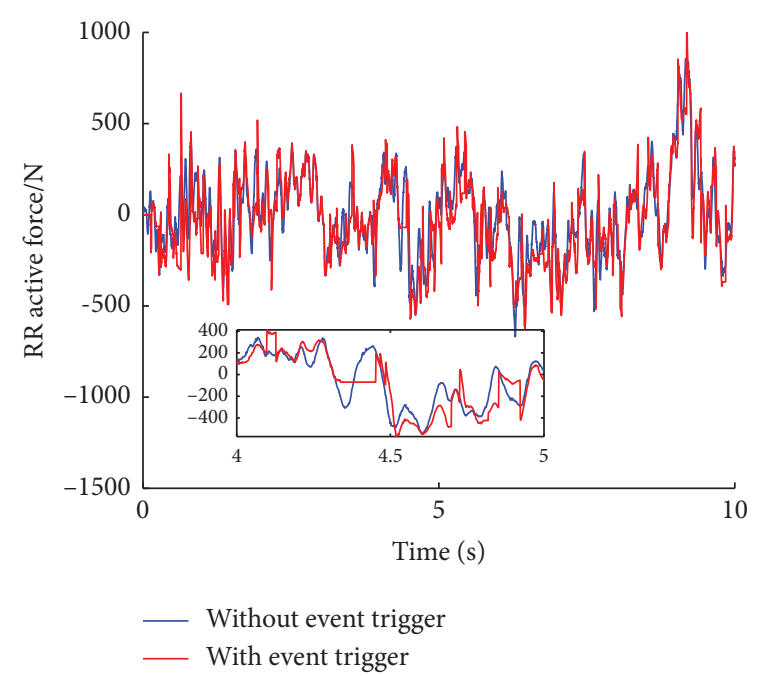

(d)

FIgURE 12: Actuator forces with the event trigger mechanism.

TABLE 3: Comparison of root mean square values.

\begin{tabular}{lccccc}
\hline & Vertical acceleration & Pitch acceleration & Roll acceleration & FL suspension deflection & FL tire deflection \\
\hline With trigger & 0.2437 & 0.3576 & 0.4565 & 0.0027 & 0.0036 \\
Without trigger & 0.2422 & 0.3559 & 0.4548 & 0.0027 & 0.0036 \\
NFTSM & 0.2330 & 0.3506 & 0.4509 & 0.0026 & 0.0037 \\
Passive & 0.4764 & 0.6427 & 0.8187 & 0.0032 & 0.0031 \\
\hline
\end{tabular}

also added to verify the effectiveness of the proposed method. As Table 3 shows, the introduction of the trigger mechanism has very little effect on the vehicle's riding comfort, and the designed controller has performance similar to that of the NFTSM controller in improving the sprung mass characteristics.

\section{Conclusions}

The aim of the present research was to deal with the quantization error and improve the actuator efficiency of the control system. A sliding-mode-based quantized feedback controller was designed for the active suspension system to improve the riding comfort with external disturbances. Then, an event trigger mechanism was introduced in the design process of the quantization controller to reduce the operation frequency of the actuator. Some conclusions can be drawn as follows:

(1) Compared with the static quantizer, the combined quantizer can achieve better performance

(2) The introduction of the trigger mechanism can effectively reduce the operating frequency of the actuator while maintaining the control effect of the active system

(3) The overall controller of the active suspension can improve riding comfort while maintaining handling stability without deterioration in the presence of external disturbances
The performance of the proposed controller has been validated under random road excitation in the MATLAB/ Simulink environment.

In the future works, the influence of an in-wheel motor on vehicle vertical vibration will be considered, and critical test conditions will be applied. Besides, the longitude and lateral influence will also be considered.

\section{Data Availability}

The data used to support the findings of this study are included within the article.

\section{Conflicts of Interest}

The authors declare no potential conflicts of interest with respect to the research, authorship, and/or publication of this article.

\section{Acknowledgments}

This research was funded by the Natural Science Foundation of Shannxi Province, grant no. 2021JQ-857, and Shaanxi Provincial Key Research and Development Project, grant no. 2020ZDLGY16-01.

\section{References}

[1] F. Zhao, S. S. Ge, F. Tu, Y. Qin, and M. Dong, “Adaptive neural network control for active suspension system with actuator 
saturation," IET Control Theory \& Applications, vol. 10, no. 14, pp. 1696-1705, 2016.

[2] J. J. Rath, M. Defoort, H. R. Karimi, and K. C. Veluvolu, "Output feedback active suspension control with higher order terminal sliding mode," IEEE Transactions on Industrial Electronics, vol. 64, no. 2, pp. 1392-1403, 2017.

[3] Y. Qin, F. Zhao, Z. Wang et al., "Comprehensive analysis for influence of controllable damper time delay on semi-active suspension control strategies," Journal of Vibration \& Acoustics, vol. 139, no. 3, 2017.

[4] H. E. Tseng and D. Hrovat, "State of the art survey: active and semi-active suspension control," Vehicle System Dynamics, vol. 53, no. 7, pp. 1-29, 2015.

[5] F. Zhao, M. Dong, Y. Qin et al., "Adaptive neural-sliding mode control of active suspension system for camera stabilization," Shock and Vibration, vol. 2015, Article ID542364, 8 pages, 2015.

[6] W. Sun, H. Gao, and O. Kaynak, "Adaptive backstepping control for active suspension systems with hard constraints," IEEE/ASME Transactions on Mechatronics, vol. 18, no. 18, pp. 1072-1079, 2013.

[7] W. Sun, Z. Zhao, and H. Gao, "Saturated adaptive robust control for active suspension systems," IEEE Transactions on Industrial Electronics, vol. 60, no. 9, pp. 3889-3896, 2013.

[8] N. Yagiz and Y. Hacioglu, "Backstepping control of a vehicle with active suspensions," Control Engineering Practice, vol. 16, no. 12, pp. 1457-1467, 2008.

[9] W. Rattasiri and S. K. Halgamuge, "Computationally advantageous and stable hierarchical fuzzy systems for active suspension," IEEE Transactions on Industrial Electronics, vol. 50, no. 1, pp. 48-61, 2003.

[10] H. Pan and W. Sun, "Nonlinear output feedback finite-time control for vehicle active suspension systems," IEEE Transactions on Industrial Informatics, vol. 15, no. 4, pp. 2073-2082, 2019.

[11] H. Pan, X. Jing, W. Sun, and H. Gao, "A bioinspired dynamics-based adaptive tracking control for nonlinear suspension systems," IEEE Transactions on Control Systems Technology, vol. 26, no. 3, pp. 903-914, 2018.

[12] B. Mohan and S. B. Phadke, "Variable structure active suspension system," in Proceeedings of the IEEE IECON, International Conference on Industrial Electronics, Control, and Instrumentation, pp. 1945-1948, Tokyo, Japan, July 1996.

[13] P. C. Chen, C. W. Chen, and W. L. Chiang, "GA-based modified adaptive fuzzy sliding mode controller for nonlinear systems," Expert Systems with Applications, vol. 36, no. 3, pp. 5872-5879, 2009.

[14] R.-J. Lian, "Enhanced adaptive self-organizing fuzzy slidingmode controller for active suspension systems," IEEE Transactions on Industrial Electronics, vol. 60, no. 3, pp. 958-968, 2013.

[15] J. Lin and R.-J. Lian, "Intelligent control of active suspension systems," IEEE Transactions on Industrial Electronics, vol. 58, no. 2, pp. 618-628, 2011.

[16] S.-J. Huang and H.-Y. Chen, "Adaptive sliding controller with self-tuning fuzzy compensation for vehicle suspension control," Mechatronics, vol. 16, no. 10, pp. 607-622, 2006.

[17] H. Pan, X. Chang, and D. Zhang, "Event-triggered adaptive control for uncertain constrained nonlinear systems with its application," IEEE Transactions on Industrial Informatics, vol. 16, no. 6, pp. 3818-3827, 2019.

[18] B.-C. Zheng and Y.-M. Xue, "A sliding sector approach to quantized feedback variable structure control," International
Journal of Control, Automation and Systems, vol. 11, no. 6, pp. 1177-1186, 2013.

[19] B.-C. Zheng and G.-H. Yang, "Quantised feedback stabilisation of planar systems via switching-based sliding-mode control," IET Control Theory \& Applications, vol. 6, no. 1, pp. 149-156, 2012.

[20] B.-C. Zheng and G.-H. Yang, "Robust quantized feedback stabilization of linear systems based on sliding mode control," Optimal Control Applications and Methods, vol. 34, no. 4, pp. 458-471, 2013.

[21] J. Lunze and D. Lehmann, "A state-feedback approach to event-based control," Automatica, vol. 46, no. 1, pp. 211-215, 2010.

[22] K. J. Aström, "Event based control," in Analysis and Design of Nonlinear Control Systems, pp. 127-147, Springer, Berlin, Germany, 2008.

[23] A. Sinha, "Temperature regulation in a Continuous Stirred Tank Reactor using event triggered sliding mode control," in Proceedings of the Ifac Third International Conference on Advances in Control and Optimization of Dynamical Systems, Hyderabad, India, July 2018.

[24] H. K. Khalil, Nonlinear Systems, Prentice-Hall, Upper Saddle River, NJ, USA, 3rd edition, 2002.

[25] ISO 8608, Mechanical Vibration-Road Surface Profiles-Reporting of Measured Data, International Standardization Organization, Geneva, Switzerland, 1995.

[26] H. Ren, S. Chen, and Z. Wu, "Model of excitation of random road profile in time domain for a vehicle with four wheels," in Proceedings of the International Conference on Mechatronic Science, Electric Engineering and Computer, pp. 2332-2335, Jilin, China, August 2011.

[27] J. Sun, J. Y. Cong, L. Gu et al., "Higher order sliding mode control for active suspension systems subject to actuator faults and disturbances," Proceedings of the Institution of Mechanical Engineers, vol. 233, no. 2, pp. 280-298, 2019. 